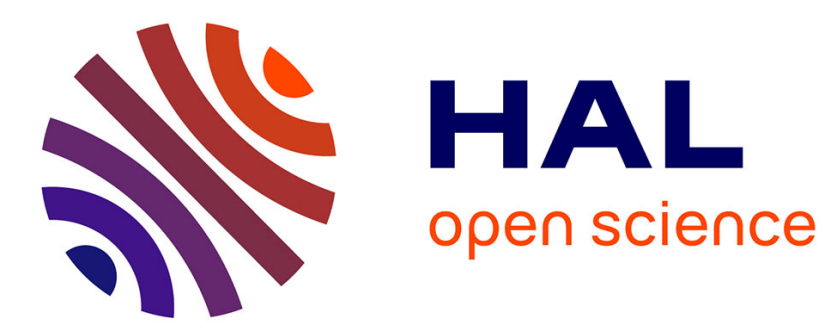

\title{
A Hierarchy of Monadic Effects for Program Verification Using Equational Reasoning
}

Reynald Affeldt, David Nowak, Takafumi Saikawa

\section{To cite this version:}

Reynald Affeldt, David Nowak, Takafumi Saikawa. A Hierarchy of Monadic Effects for Program Verification Using Equational Reasoning. Mathematics of Program Construction - 13th International Conference, MPC 2019, Porto, Portugal, October 7-9, 2019, Oct 2019, Porto, Portugal. pp.226-254, 10.1007/978-3-030-33636-3_9. hal-02359796

\section{HAL Id: hal-02359796 https://hal.science/hal-02359796}

Submitted on 25 Nov 2020

HAL is a multi-disciplinary open access archive for the deposit and dissemination of scientific research documents, whether they are published or not. The documents may come from teaching and research institutions in France or abroad, or from public or private research centers.
L'archive ouverte pluridisciplinaire HAL, est destinée au dépôt et à la diffusion de documents scientifiques de niveau recherche, publiés ou non, émanant des établissements d'enseignement et de recherche français ou étrangers, des laboratoires publics ou privés. 


\title{
A Hierarchy of Monadic Effects for Program Verification using Equational Reasoning ${ }^{\star}$
}

\author{
Reynald Affeldt ${ }^{[0000-0002-2327-953 X] 1}$, David Nowak ${ }^{2}$, and Takafumi \\ Saikawa ${ }^{[0000-0003-4492-745 X] 3}$ \\ ${ }^{1}$ National Institute of Advanced Industrial Science and Technology, Japan \\ ${ }^{2}$ CRIStAL $^{\star \star}$, France \\ ${ }^{3}$ Nagoya University \& Peano System Inc., Japan
}

\begin{abstract}
One can perform equational reasoning about computational effects with a purely functional programming language thanks to monads. Even though equational reasoning for effectful programs is desirable, it is not yet mainstream. This is partly because it is difficult to maintain pencil-and-paper proofs of large examples. We propose a formalization of a hierarchy of effects using monads in the Coq proof assistant that makes equational reasoning practical. Our main idea is to formalize the hierarchy of effects and algebraic laws like it is done when formalizing hierarchy of traditional algebras. We can then take advantage of the sophisticated rewriting capabilities of Coq to achieve concise proofs of programs. We also show how to ensure the consistency of our hierarchy by providing rigorous models. We explain the various techniques we use to formalize a rich hierarchy of effects (with nondeterminism, state, probability, and more), to mechanize numerous examples from the literature, and we furthermore discuss extensions and new applications.
\end{abstract}

\section{Introduction}

Our goal is to provide a framework to produce formal proofs of semantical correctness for programs with effects. To formalize effects, we use monads. The notion of monad is one of the category-theoretic frameworks that are used to formalize effects in programming languages and reason about them. It is not the only available option for this purpose (for example, algebraic effects provide an alternative [36, $\S 5]$ ), but monads comparatively have a longer history in proving themselves useful for the study of semantics [28] as well as for actual programming languages like Haskell as a construct to represent effects 40. Though there exist a few formalizations of monads in proof assistants, they do not support well our interest in proving programs. Existing formalizations often focus on category theory [17/39] or on meta-theory of programming languages 9]. In

* This is a preprint of a paper to be presented at MPC 2019 (http://www.cs.nott.ac.uk/ ${ }^{\sim p s z g m h / m p c 19 . h t m l) ~}$

** Univ. Lille, CNRS, Centrale Lille, UMR 9189 - CRIStAL - Centre de Recherche en Informatique Signal et Automatique de Lille, F-59000 Lille, France 
contrast, proving programs raises specific practical challenges, among which the generic problem of combining monads is a central issue.

In the practical use-cases of monads in programming, a programmer often has to combine two or more monads in order to deal with several effects in the same context. The combination of monads can be carried out in an ad-hoc way [22] There exist more generic ways to combine monads under specific conditions [21] including the distributive law between monads, which are unfortunately not always satisfied [38 and therefore do not provide a practical solution.

In this paper, we propose a formalization of monads in the CoQ proof assistant that addresses monad combination in a practical way. The main idea is to favor a good representation of the hierarchy of effects and their equational theory in terms of interfaces. In other words, monads are composed as in Haskell. We insist on interfaces but this does not preclude the formal construction of models: they just come afterwards. It happens that this corresponds to the presentation of monads as used in monadic equational reasoning [13, so that a direct consequence of our approach is that we can reproduce formally and faithfully pencil-and-paper proofs from the literature.

When it comes to proving properties of effectful programs, there is more than the hierarchy of effects: one also needs to provide practical tools to perform equational reasoning. With this respect, the second aspect of our approach is to leverage the rewriting capabilities of COQ by favoring a shallow embedding. Shallow embedding is a well-known encoding technique through which one can reuse the native language of the proof assistant at hand. This bears the promise of a reduced formalization effort and it indeed experimentally met some success [16|20] (formal verification using a shallow embedding often relies on a combination of monads and Hoare logic, e.g., [19]). However, most formal verification frameworks proceeds via a deep embedding of the target language, which requires substantial instrumentations of syntax and semantics, resulting in technical lemmas that are difficult to use, which in turn call for meta-programming. Though this paper favors shallow embedding, it does not prevent syntactical reasoning, as we will demonstrate.

Our main contribution in this paper is to demonstrate a combination of formalization techniques that make formal reasoning about effectful programs in CoQ practical:

- We formalize a rich hierarchy of effects (failure, exception, nondeterminism, state, probability, and more) whose heart is the theory by Gibbons and Hinze [13] that we extend with more monads and formal models. The key technique is packed classes [11, a methodology used in the МАтнСомР library [26] to formalize the hierarchy of mathematical structures. We do not know of another mechanization with that many monads.

- We provide many definitions and lemmas that allow for the mechanization of several examples. Because we use a shallow embedding, we can leverage CoQ native rewriting capabilities, in particular SSREFLECT's rewrite tactic 15.

- The proof scripts we obtain are faithful to the original proofs. We benchmark our library against numerous examples of the literature (most examples 
from [12]13 30[31]) and observe that formal proofs closely match their penciland-paper counterparts and that they can actually be shorter thanks to the terseness of SSREFLECT's tactic language. We also apply our framework to new examples such as the formalization of the semantics of an imperative language.

Outline. In Sections 2 and 3 , we show how we build a hierarchy of algebraic laws on top of the theory of monads. In Sect. 4, we illustrate its usability for mechanizing pencil-and-paper proofs. We then deal with syntactic properties in Sect. 5. In Sect. 66, we show how we can give models to our algebraic laws, thus ensuring their consistency. In Sect. 7, we discuss some technical aspects of our formalization of monads that are specific to CoQ. We finally discuss related work in Sect. 8 before concluding in Sect. 9

\section{Build a Hierarchy of Algebraic Laws on Top of the Theory of Monads}

The heart of our formalization is a hierarchy of effects. Each effect is represented by a monad with some additional algebraic structure that defines the effect, providing effect operators and equations that capture the properties of operators. These effects form a hierarchy in the sense that each effect is the result of a series of extensions starting from the theory of functors, each step extending an existing one in such a way that it shares operators and properties with its parents. We use the methodology of packed classes, which was originally used to formalize mathematical structures [11. We explain how we use packed classes to formalize monads in Sect.2.1 and to combine monads in Sect. 2.2. The next section makes a thorough presentation of the complete hierarchy (depicted in Fig. 1).

\subsection{Basic Layers: Theories of Functors and Monads}

Our formalization of monads starts with a formal definition of functors. This is in contrast to the hierarchy from Gibbons and Hinze [13, where the monad's functor action on morphisms (fmap) is defined using bind (hereafter, we use the infix notation $\gg=$ for bind); starting with functors simplifies the organization of lemmas used in monadic equational reasoning and results in a more robust hierarchy.

Functors as the Base Packed Class. The class of functors is defined in the module Functor below. The definition follows the usual one in category theory 25 except that the domain and codomain of functors are fixed to Type. In set-theoretical semantics, Type is interpreted as the universe of sets, thus rendering our functors to be the endofunctors on the category Set of sets and functions.

We use CoQ modules only to get a namespace. Inside this namespace, functors are defined by the dependent record class_of with one field $f$ satisfying the 
functor laws (the naming should be self-explanatory, see Table 2 , Appendix B in case of doubt). The type of functors $t$ is a dependent record ${ }^{4}$ with a function $m$ of type Type $\rightarrow$ Type, which is the object part of the functor, that satisfies the class_of interface. The morphism part appears as $f$ in the record. We define Fun to refer to it, but the purpose of the definition is essentially technical. It does not reduce (thanks to the simpl never declaration) and can therefore be used to provide a stable notation: $\mathrm{F} \# \mathrm{~g}$ denotes the action of a functor $\mathrm{F}$ on a function $\mathrm{g}$. Last, we provide a notation functor that denotes the type Functor.t outside of the module and a coercion so that functors can be used as if they were functions (by taking the first projection $\mathrm{m}$ of the dependent record that represents their type).

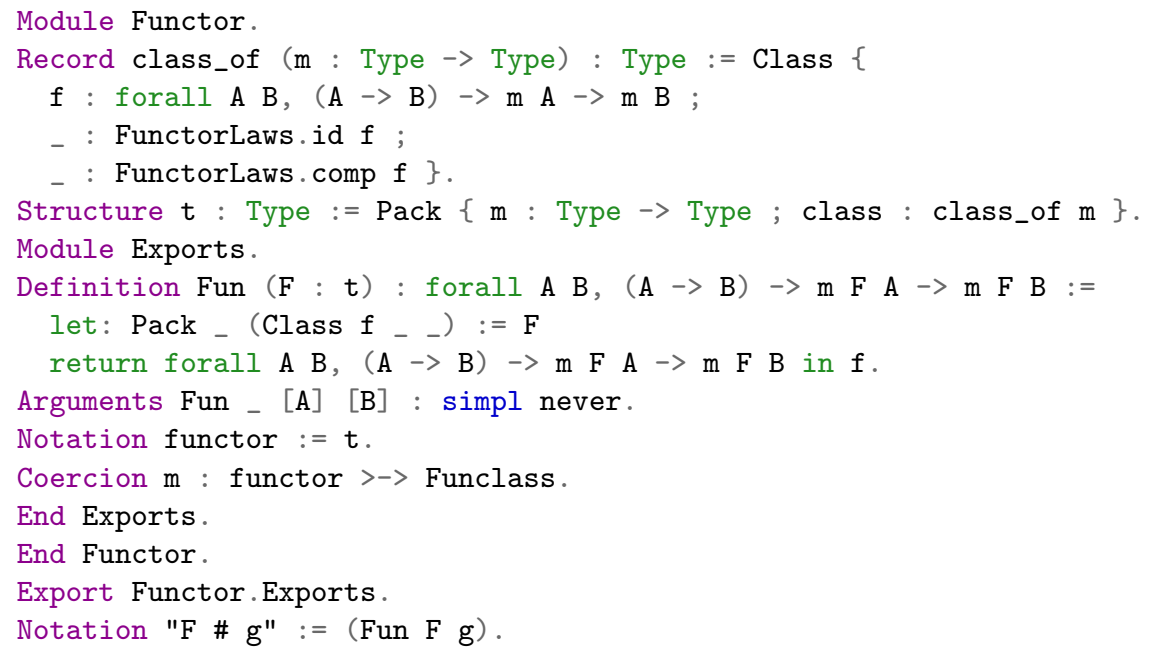

Monads as a Packed Class Extension. A monad in category theory is defined as an endofunctor $M$ with two natural transformations $\eta:$ Id $\rightarrow M$ (where Id is the identity endofunctor) and $\mu: M^{2} \rightarrow M$ satisfying some laws [25]. Following the above definition, our class of monads is defined as an extension of the class of functors.

Inside the module Monad below, the interface of monads is captured by the dependent record mixin_of with two fields ret and join, that correspond to $\eta$ and $\mu$ respectively, satisfying the monad laws (Table 2, Appendix B). The type of monads Monad.t is a dependent record with a function Monad.m of type Type $\rightarrow$ Type that satisfies a class_of interface; the latter extends the class of functors (its base) with the mixin of monads. Thanks to the definition baseType, a monad can also be seen as a functor. This fact is handled transparently by the type system of CoQ thanks to the Canonical command.

Module Monad

Record mixin_of (M : functor) : Type := Mixin \{

${ }^{4}$ Record and Structure are synonymous but the latter is used to emphasize that it is to be made Canonical. 


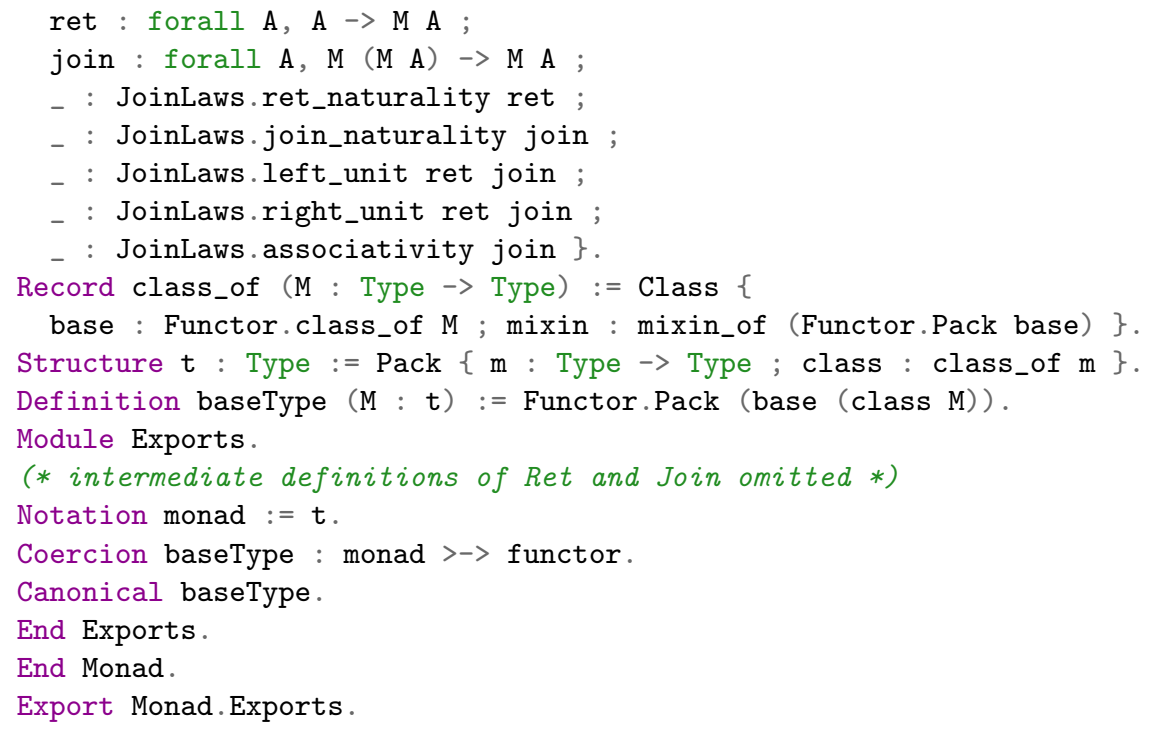

The monad above is defined in terms of ret and join. In programming, the operator bind is more common. Using CoQ notation, its type can be written forall A B, M A $\rightarrow($ A $\rightarrow$ M B) $\rightarrow$ M B. The second argument of type A $\rightarrow$ M B is a CoQ function that represents a piece of effectful program. This concretely shows that we are heading for a framework using a shallow embedding. We provide an alternative way to define monads using ret and bind. Let us assume that we are given ret and bind functions that satisfy the monad laws:

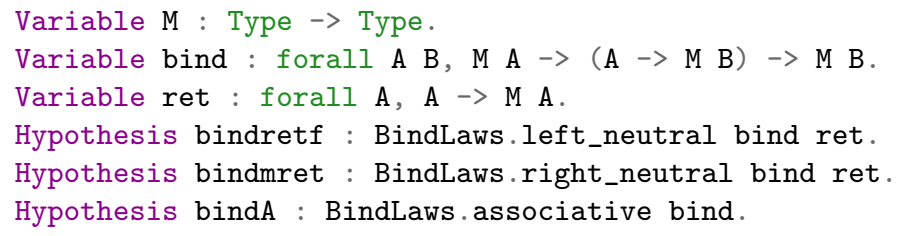

We can then define fmap that satisfies the functor laws:

Definition fmap A B ( $f: A \rightarrow B)(m: M A):=\operatorname{bind} m(\operatorname{ret}(A:=B) \backslash 0 f)$.

Lemma fmap_id : FunctorLaws.id fmap.

Lemma fmap_o: FunctorLaws.comp fmap.

We can use these lemmas to build $M^{\prime}$ of type functor and use $M^{\prime}$ to define join:

Definition join A (pp : $\left.M^{\prime}\left(M^{\prime} A\right)\right):=$ bind pp id.

It is now an exercise to prove that ret and join satisfy the monad laws, using which we eventually build $\mathrm{M}$ of type monad. We call Monad_of_ret_bind this construction that we use in the rest of this paper. 


\subsection{Extensions: Specific Monads as Combined Theories}

In the previous section, we explained the case of a simple extension: one structure that extends another. In this section we explain how a structure extends two structures. Here, we just explain how we combine theories, how we provide concrete models for combined theories is the topic of Sect. 6.

For the sake of illustration, we use the nondeterminism monad that extends both the failure monad and the choice monad. The failure monad failmonad extends the class of monads (Sect. 2.1) with a failure operator fail that is a left-zero of bind. Since the extension methodology is the same as in Sect. 2.1. we provide the code with little explanation 5

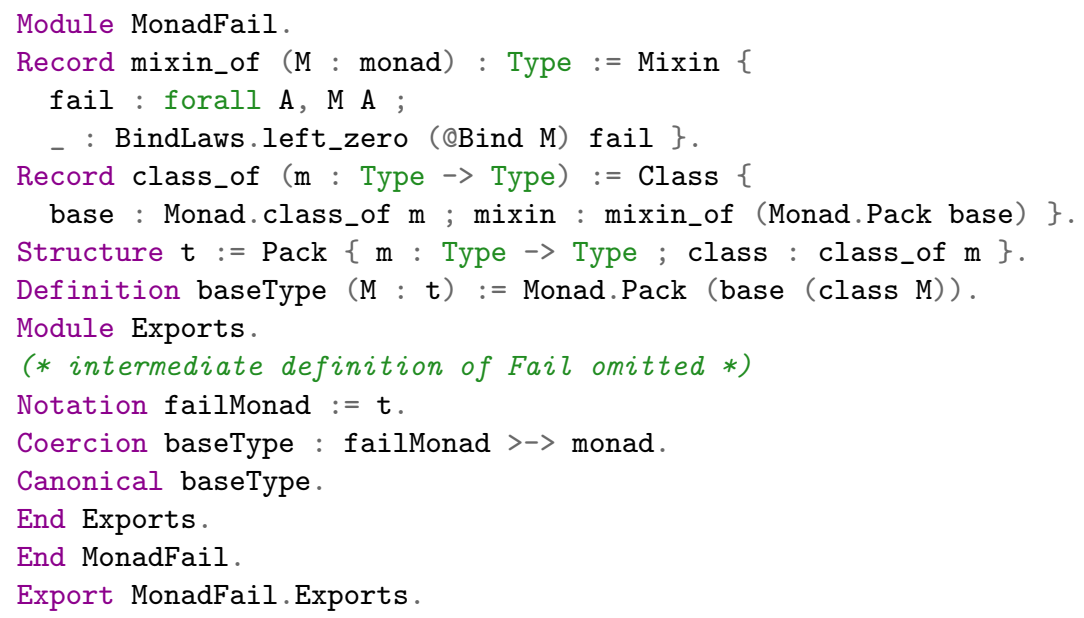

The choice monad altMonad extends the class of monads with a choice operator alt (infix notation: $[\sim]$; prefix: $[\sim p]$ ) that is associative and such that bind distributes leftwards over choice (the complete code is displayed in Appendix A).

The nondeterminism monad nondetMonad defined below extends both the failure monad and the choice monad. This extension is performed by first selecting the failure monad as the base whose base itself is further required to satisfy the mixin of the choice monad (see base2 below). As a result, a nondeterminism monad can be regarded both as a failure monad (definition baseType) or as a choice monad (definition alt_of_nondet): both views are declared as Canonical.

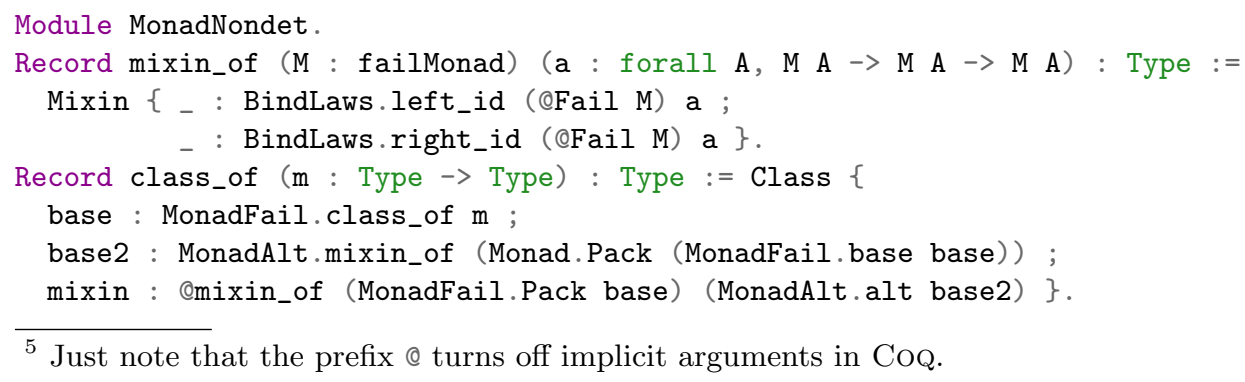

${ }^{5}$ Just note that the prefix @ turns off implicit arguments in CoQ. 


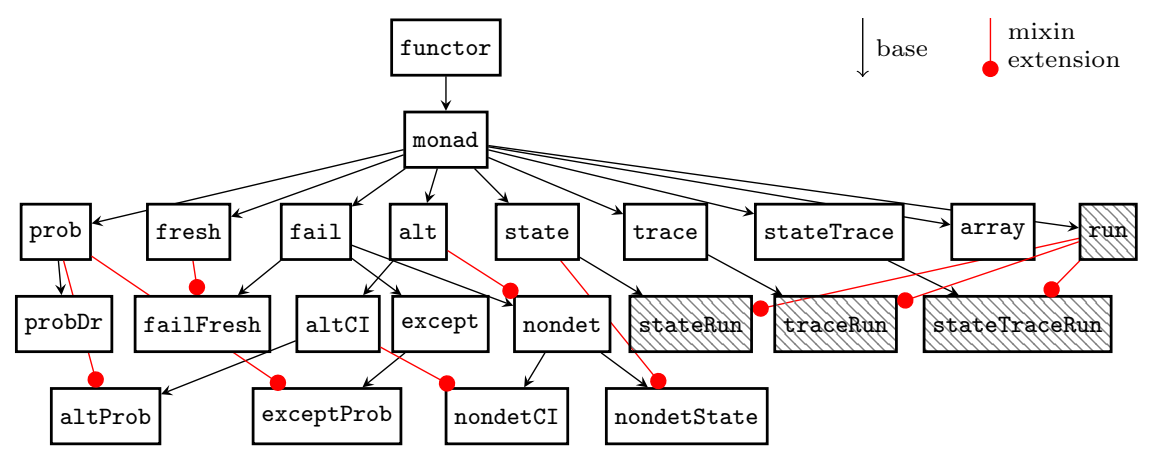

Fig. 1. Hierarchy of effects formalized. See Table 3 for the algebraic laws. In the CoQ scripts [3], the monad xyz appears as xyzMonad.

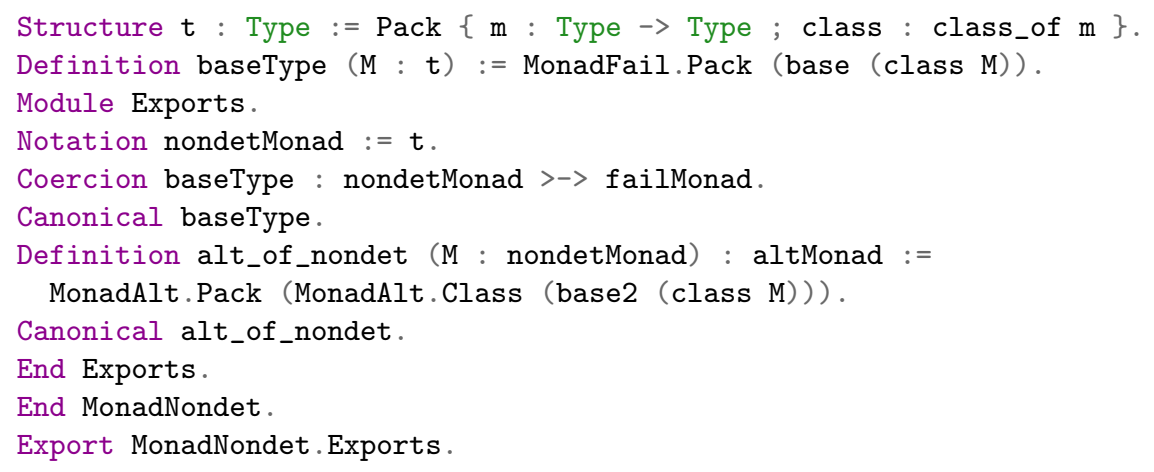

\section{More Monads from our Hierarchy of Effects}

This section complements the previous one by explaining more monads from our hierarchy of effects (Fig. 1). We explain these monads in particular because they are used later in the paper ${ }^{6}$ They are all obtained using the combination technique previously explained in Sect. 2.2 .

\subsection{The Exception Monad}

The exception monad exceptMonad extends the failure monad (Sect. 2.2) with a Catch operator with monoidal properties (the Fail operator being the neutral) and the property that unexceptional bodies need no handler [13, §5]:

\footnotetext{
${ }^{6}$ The exception monad is used in the motivating example of Sect. 4.1. state-related monads are used in particular to discuss the relation with deep embedding in Sect. 5.1 the state-trace monad is used in the application of Sect. 5.2 and a model of the probability monad is provided in Sect. 6.2 .
} 


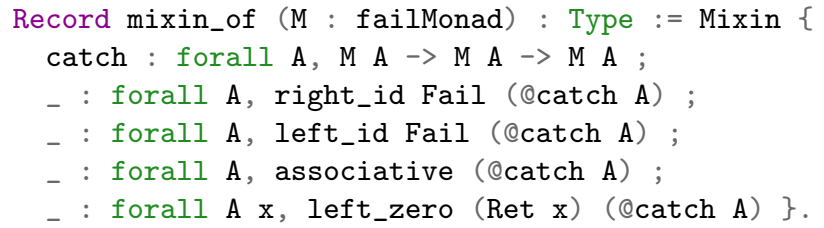

The algebraic laws are given self-explanatory names; see Table 1. Appendix B in case of doubt.

\subsection{The State Monad and Derived Structures}

The state monad is certainly the first monad that comes to mind when speaking of effects. It denotes computations that transform a state (type $\mathrm{S}$ below). It comes with a Get operator to yield a copy of the state and a Put operator to overwrite it. These functions are constrained by four laws [13]:

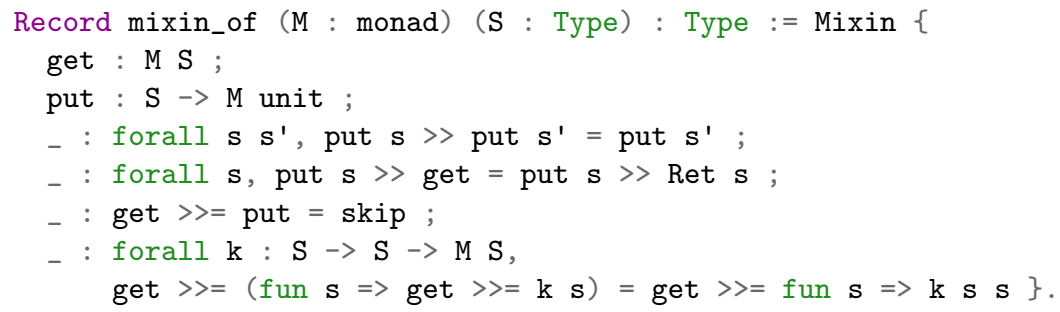

Reification of State Monads. We introduce a Run operator to reify staterelated monads (this topic is briefly exposed in [13, $\S 6.2$ ], we use reification in Sect. 3.3). First, the operator run defines the semantics of Ret and Bind according to the following equations:

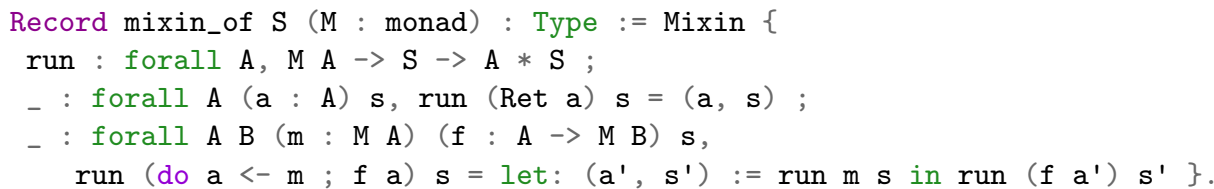

The type of run shows that it turns a state into a pair of a value and a state. We call the monad that extends monad with such an operator a runMonad. Second, we combine stateMonad with runMonad and extend it with Run equations for Get and Put; this forms the stateRunMonad:

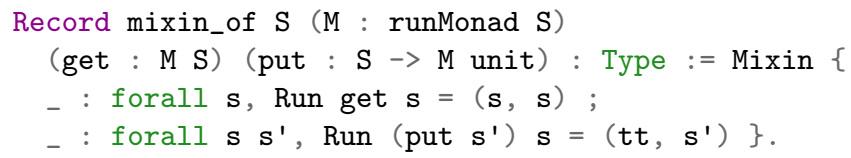

Monads with the Run operator appear shaded in Fig. 1, they can be given concrete models so as to run sample programs inside CoQ (there are toy examples in [3, file smallstep_examples.v]). 
The Backtrackable-state Monad. The monad nondetStateMonad combines state with nondeterminism (recall that the nondeterminism monad is itself already the result of such a combination) and extends their properties with the properties of backtrackable-state ([13, §6], [30, §4]):

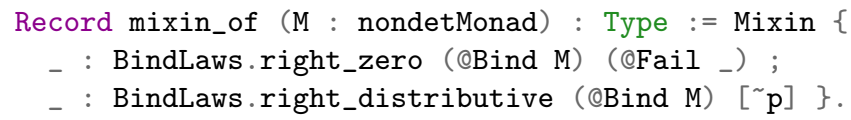

Failure is a right zero of composition to discard any accumulated stateful effects and composition distributes over choice.

\subsection{The State-trace Monad}

The state-trace monad is the result of combining a state monad with a trace monad. Our trace monad extends monads with a Mark operator to record events:

Record mixin_of $\mathrm{T}$ ( $\mathrm{m}$ : Type $\rightarrow$ Type) : Type :=

Mixin $\{\operatorname{mark}: \mathrm{T} \rightarrow \mathrm{m}$ unit $\}$.

We call the operators of the state-trace monad st_get, st_put, and st_mark (notations: stGet, stPut, stMark). stGet and stPut fulfill laws similar to the ones of Get and Put, but their interactions with stMark call for two more laws:

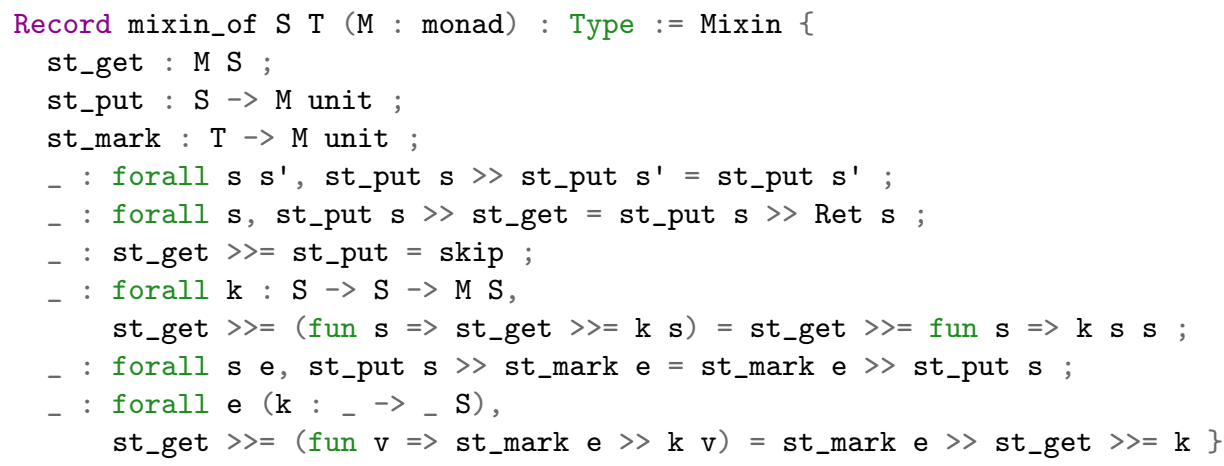

\subsection{The Probability Monad}

First, we define a type prob of probabilities [4] as reals of type $\mathrm{R}$ between 0 and 1 :

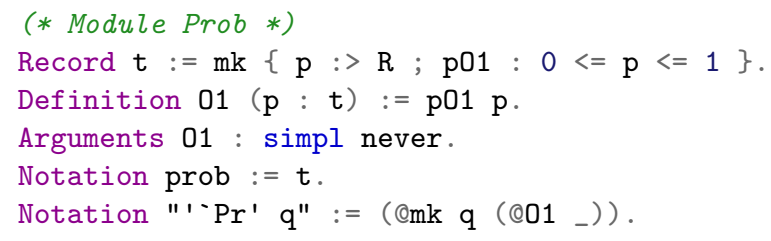


This definition is interesting because the notation makes it possible to write concrete probabilities succinctly: the proof that the real is between 0 and 1 is hidden and can be inferred automatically. For example, the probability $\frac{1}{2}$ is written ${ }^{-} \operatorname{Pr} / 2$, the probability $\bar{p}=1-p$ (where $p$ is a probability) is written - Pr p. ${ }^{\sim}$, etc. This is under the condition that we equip COQ with appropriate canonical structures. For example, here follows the registration of the proof $0 \leq$ $\frac{1}{p} \leq 1$ that makes it possible to write ${ }^{-} \operatorname{Pr} / 2$ (IZR injects integers into reals):

Lemma prob_IZR ( $\mathrm{p}$ : positive) : $0<=/$ IZR (Zpos p) $<=1$.

Canonical probIZR ( $\mathrm{p}$ : positive) $:=$ @Prob.mk _ (prob_IZR p).

The above type and notation for probabilities lead us to the following mixin for the probability monad [13, $\S 8]$ :

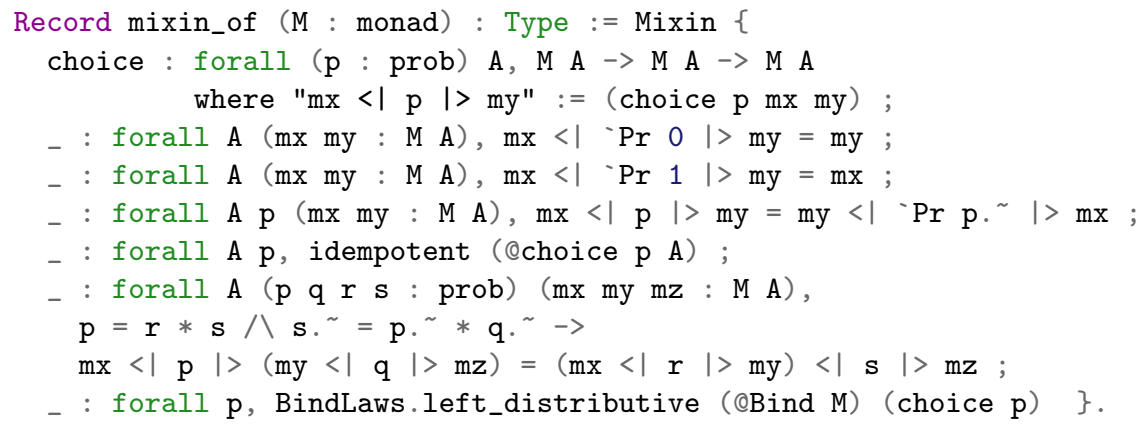

$\mathrm{mx}\langle\mathrm{p}>\mathrm{my}$ behaves as $\mathrm{mx}$ with probability $\mathrm{p}$ and as my with probability $\bar{p}$. Lines 6 and 7 are a skewed commutativity law and idempotence. Lines 810 is a quasi associativity law. Above laws are the same as convex spaces [18, Def 3]. Line 11 says that bind left-distributes over probabilistic choice.

\subsection{Other Monads in the Hierarchy of Effects}

Figure 1 pictures the hierarchy of effects that we have formalized; Table 3 (Appendix C lists the corresponding algebraic laws. The starting point is the hierarchy of [13. It needed to be adjusted to fit other papers [1|12]30|31]:

- As explained in Sect. 2.1. we put functors at the top to simplify formal proofs.

- The examples of [13] relying on nondeterministic choice use altMonad. However, the combination of nondeterminism and probability in altProbMonad requires idempotence and commutativity of nondeterministic choice [12]. Idempotence and commutativity are also required in the first part of [31]. We therefore insert the monad altCIMonad with those properties in the hierarchy, and also the monad nondetCIMonad to deal more specifically with the second part of 31.

- The probability monad probMonad is explained in Sect. 3.4. The probability monad probDrMonad is explained in [13, §8]. The main difference with [13. is that we extract probMonad from probDrMonad as an intermediate step. 
probDrMonad extends probMonad with right distributivity of bind $(\cdot \gg=\cdot)$ over probabilistic choice $(\cdot \triangleleft \cdot \triangleright \cdot)$. The reason is that this property is not compatible with distributivity of probabilistic choice over nondeterministic choice $(\cdot \square \cdot)$ and therefore needs to be put aside to be able to form altProbMonad by combining probMonad and altMonad (the issue is explained in [1]).

There are two more monads that we have not explained. exceptProbMonad combines probability and exception [12, §7.1]. freshMonad and failFreshMonad are explained in [13, §9.1]; freshMonad provides an operator to generate fresh labels.

We have furthermore extended the hierarchy of [13] with reification (Sect. 3.2, the trace and state-trace monads (Sect. 3.3), and the array monad [35].

\section{Monadic Equational Reasoning}

The faithful mechanization of pencil-and-paper proofs by monadic equational reasoning is the main benefit of a hierarchy of effects built with packed classes. After a motivating example in Sect.4.1, we explain how the CoQ rewrite tactics copes with notation and lemma overloading in Sect. 4.2 . Section 4.3 explains the technical issue of rewriting under function abstractions. Section 4.4 provides an overview of the existing proofs that we have mechanized.

\subsection{Motivating Example: the Fast Product}

This example shows the equivalence between a functional implementation of the product of integers with a monadic version (fastprod) [13. On the left of Fig. 2 we (faithfully) reproduce the series of rewritings that constitute the original proof. On the right, we display the equivalent series of CoQ goals and tactics.

The product of natural numbers is simply defined as foldr muln 1. A "faster" product can be implemented using the failure monad (Sect. 2.2) and the exception monad (Sect. 3.1):

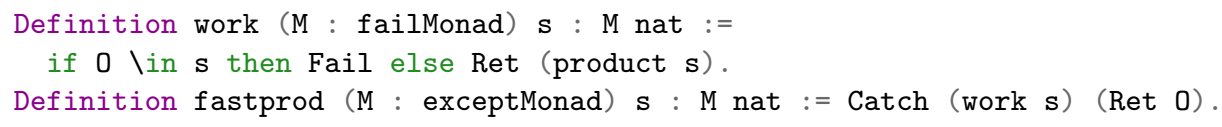

We observe that the user can write a monadic program with one monad and use a notation from a monad below in the hierarchy. Concretely, here, work is written with failMonad but still uses the unit operator Ret of the base monad. The same can be said of fastprod. This is one consequence of packed classes. What happens is that CoQ inserts appropriate calls to canonical structures so that the program type-checks. In fact, the program work and fastprod are actually equal to the following (more verbose) ones:

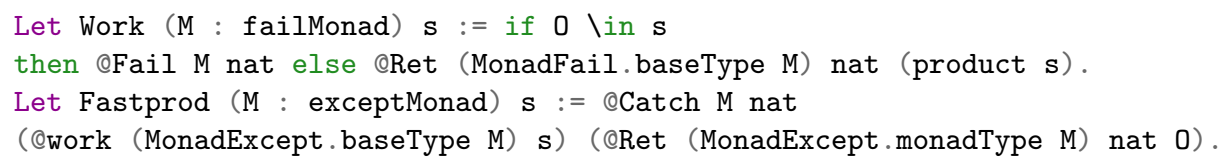




\begin{tabular}{|c|c|}
\hline Pencil-and-paper proof [13, §5.1] & termediate goals and tactics \\
\hline fastprod xs & fastprod s \\
\hline$=\llbracket$ definition of fastprod $\rrbracket$ & $=\llbracket$ rewrite /fastprod $\rrbracket$ \\
\hline catch (work xs) (ret 0) & Catch (work s) (Ret 0) \\
\hline$=\llbracket$ specification of work $\rrbracket$ & $=\llbracket$ rewrite /work $\rrbracket$ \\
\hline catch (if 0 in $x s$ then fail & Catch (if 0 \in $s$ then Fail \\
\hline else ret (product $x s))($ ret 0$)$ & else Ret (product $\mathbf{s}))(\operatorname{Ret} 0)$ \\
\hline$=\llbracket$ lift out the conditional $\rrbracket$ & $=\llbracket$ rewrite lift_if if_ext $\rrbracket$ \\
\hline if 0 in $x s$ then catch fail (ret 0 ) & if 0 \in $\mathrm{s}$ then Catch Fail (Ret 0 ) \\
\hline else catch $($ ret $($ product $x s))($ ret 0$)$ & else Catch (Ret (product s)) (Ret 0) \\
\hline$=\llbracket$ laws of catch, fail, and ret $\rrbracket$ & $=\llbracket$ rewrite catchfailm catchret $\rrbracket$ \\
\hline if 0 in $x s$ the & if $0 \backslash$ \in $\mathrm{s}$ then $\operatorname{Ret} 0$ \\
\hline else $\mathrm{r}$ & else Ret (product s) \\
\hline$=\llbracket \mathrm{a}$ & $=\llbracket$ case $: i$ \\
\hline if & $\forall s .0 \in s$ \\
\hline & \\
\hline & $=\llbracket$ move \\
\hline & Ret (product $s$ ) \\
\hline
\end{tabular}

Fig. 2. Comparison between an existing proof and our CoQ formalization

The CoQ proof that fastprod is pure, i.e., that it never throws an unhandled exception, can be compared to its pencil-and-paper counterpart in Fig. 2, Both proofs are essentially the same, though in practice the COQ proof will be streamlined in two lines (of less than 80 characters) of script:

Lemma fastprodE $\mathbf{s}$ : fastprod $\mathbf{s}=\operatorname{Ret}$ (product $\mathbf{s}$ ).

Proof.

rewrite/fastprod/work lift_if if_ext catchfailm. by rewrite catchret; case: ifPn $=>/ /$ /product $0<-$.

Qed.

The fact that we achieve the same conciseness as the pencil-and-paper proof is not because the example is simple: the same can be said of all the examples we mechanized (see Sect. 4.4).

\subsection{Basics of Equational Reasoning with Packed Classes}

Packed classes not only allow sharing of notations but also sharing of lemmas: one can rewrite a monadic program with any algebraic law from structures below in the hierarchy of effects. SSREFLECT's advanced rewrite tactic 7 becomes available to faithfully reproduce monadic equational reasoning.

For illustration, let us consider a function that nondeterministically builds a subsequence of a list using the choice monad [12, §3.1]:

\footnotetext{
7 SSREFLECT extends COQ's rewrite with contextual patterns, unfolding, etc. 15. The main benefit is that semantically-close actions can be performed on the same line of script, instead of having to interleave with other CoQ tactics such as pattern or unfold.
} 


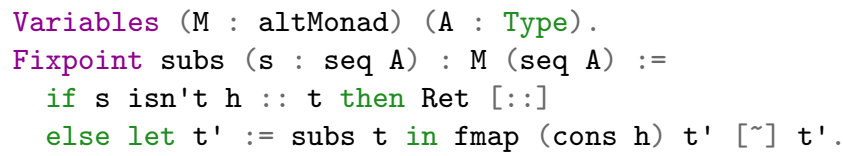

The mixed use of algebraic laws from various monads can be observed when proving that subsequences of concatenation are concatenations of subsequences:

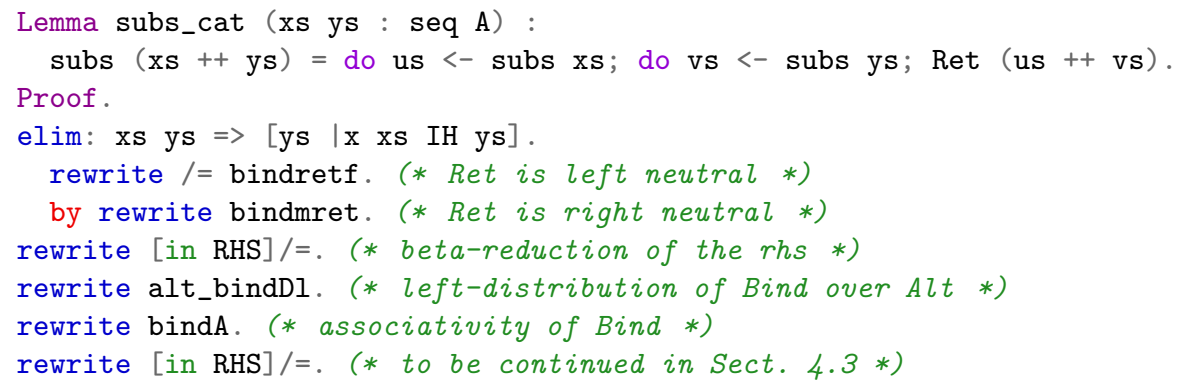

The proof is by induction on the sequence xs (line 4 ). While the lemma alt_bindD1 (line 8 belongs to the interface of the altMonad interface, the lemma bindA (line 9) comes from the monad interface.

\subsection{Rewriting under Function Abstractions}

In pencil-and-paper proofs of monadic equational reasoning, whether rewriting occurs under a function abstraction or not does not make any difference. We need custom automation to support this feature in CoQ which does not natively perform rewriting in this situation.

The proof from the previous section led us to the following subgoal:

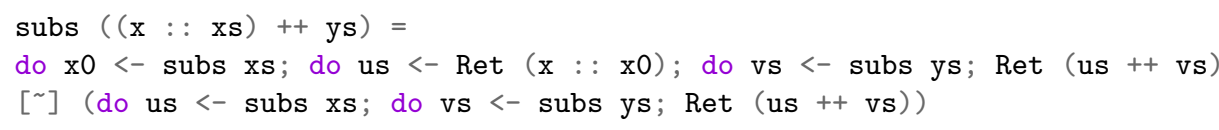

We want to turn the first branch of the nondeterministic choice

do $\mathrm{x} 0<-$ subs $\mathrm{xs} ;$ do us <- Ret $(\mathrm{x}:: \mathrm{x} 0)$; do vs <- subs ys; Ret (us ++ vs) into

do $\mathrm{x} 0<-$ subs $\mathrm{xs} ;$ do $\mathrm{vs}<-$ subs ys; $\operatorname{Ret}(\mathrm{x}:: \mathrm{x} 0++\mathrm{vs})$

but since the occurrence of Ret of interest is under the binder "do $\mathrm{x} 0<-$ ", rewrite bindretf fails. Instead, we "open" the continuation with a custom tactic Open ( $\mathrm{X}$ in subs $\mathrm{xs}>>=\mathrm{X}$ ) to get a new subgoal

do us <- Ret (x :: $\mathrm{x} 0$ ); do vs <- subs ys; Ret (us ++ vs) = ?g $\mathrm{x} 0$

where ? $\mathrm{g}$ is an existential variable. Now, rewrite bindretf succeeds:

do vs <- subs ys; $\operatorname{Ret}((\mathrm{x}:: \mathrm{x} 0)++\mathrm{vs})=? \mathrm{~g} \times \mathrm{x} 0$ 
Yet, the last Ret is still under a binder. We could again "open" the continuation but instead we use a custom "rewrite under" tactic rewrite_ cat_cons to get:

do $\mathrm{x} 1<-$ subs $\mathrm{ys} ; \operatorname{Ret}(\mathrm{x}:: \mathrm{x} 0++\mathrm{x} 1)=? \mathrm{~g} \mathrm{x} 0$

Now we can trigger unification to instantiate the existential variable and thus complete the intended rewriting.

In practice, there is little need for Open and most situations can be handled directly without revealing the existential variable using rewrite_. We chose to explain Open here because it shows how rewrite_ is implemented.

\subsection{Mechanization of Existing Pencil-and-paper Proofs}

We used our framework to mechanize the definitions, lemmas, and examples from [13] (except Sect. 10.2), from [12] (up to Sect. 7.2, which overlaps and complements [13]), examples from 3031, and examples from [21] (up to Sect. 3). This includes in particular:

- Spark aggregation: Spark is a platform for distributed computing, in which the aggregation of data is therefore nondeterministic. Monadic equational reasoning can be used to sort out the conditions under which aggregation is actually deterministic [31, $\S 4.2]$ as well as other properties. We have mechanized these results [3, file example_spark.v], which are part of a larger specification [6].

- The $n$-queens puzzle: This puzzle is used to illustrate the combination of state and nondeterminism. We have mechanized the relations between functional and stateful implementations [13, §6-7] [3, file example_nqueens.v], as well as the derivation of a version of the algorithm using monadic hylo-fusion 30 , $\S 5]$. This example demonstrates the importance of commutativity lemmas, calling for syntax reflection (see Sect. 5).

- The Monty Hall problem: We have mechanized the probability calculations for several variants of the Monty Hall problem [12/13] using probMonad, altProbMonad, and exceptProbMonad [3, file example_monty.v].

- The tree relabeling example: This example originally motivated monadic equational reasoning [13]. It amounts to show that the labels of a binary tree are distinct when the latter has been relabeled with fresh (see freshMonad) labels. We have mechanized this result [3, file example_relabeling.v].

- The swap construction: This is an example of monad composition [21]. Strictly speaking, this is not monadic equational reasoning: formalization does not require a mechanism such as canonical structures. Yet, our framework proved adequate because it allows to mix in a single equation different ret's and join's without explicit mention of which monad they belong to; inference is automatic thanks to coercions.

The level of details provided by the authors using monadic equational reasoning is helpful and provides a way to check that our mechanization is faithful. Among the differences between pencil-and-paper and mechanized proofs, 
the main one is maybe function termination. Pencil-and-paper proofs assume Haskell and do not require particular care about function termination, whereas CoQ functions must terminate, so that formalization requires an extra effort. See for example the formalization of unfoldm and hylom [3] which are not structurally terminating. These difficulties are known 32 and can be addressed using standard techniques. Another difference is that CoQ functions must be total, so that some Haskell functions cannot be formalized as such (e.g., foldr1).

We discovered a few problems in the work we have formalized. The main one was an error in a proof of monadic hylo-fusion for the $n$-queens puzzle from a draft paper 29] which has been reported to the author and fixed [30. In shor 8 , the functional specification of the $n$-queens puzzle can be rewritten using nondetStateMonad as

Get $>>=$ (fun ini $=>\operatorname{Put~}(0,[::],[::])>>$

queensBody (map Z_of_nat (iota 0 n)) $>>=$ overwrite ini)

in which queensBody can be rewritten as

hyloM (@opdot_queens M) [::] (@nilp _)

select seed_select (@well_founded_size__)

The heart of this last step was a theorem [29, Thm 4.2] (now [30, Thm 5.1]) whose hypotheses did not properly match the ones available in the course of the proof. However, we were able to complete the proof with a variant of the theorem in question. Other problems were at the level of typos (they could be easily caught by type-checking): almost none in [13, a few in the appendices of [6] (whose mechanization has not been completed yet).

\section{Properties Proved using Syntax}

Our formalization is a shallow embedding: a monadic program is a CoQ function of return-type M A for some monad M and some type A. This is practical because we can use the CoQ language to write, execute, and prove programs. However, it happens that some properties require an explicit syntax to be proved. In this section, we show how to handle such situations. The basic idea is to locally restrict programs to a subset characterized by a deep embedding. Section 5.1 is an example of property of backtrackable-states. Section 5.2 is an example of equivalence between an operational and a denotational semantics, the latter being given by a monad.

\subsection{The Commutativity of State and Nondeterminism}

The commutativity of state and nondeterminism is an important aspect of backtrackable-states [30]. Such a property can be proved directly on specific

\footnotetext{
${ }^{8}$ We just show the main steps of the derivation, we cannot reproduce all the definitions for lack of space, see the source code [3] for all the details.
} 
programs using their semantics but it can also be proved more generally using syntax.

The following predicate [30, Def 4.2] defines the commutativity of two computations $\mathrm{m}$ and $\mathrm{n}$ (in the same monad $\mathrm{m}$ ):

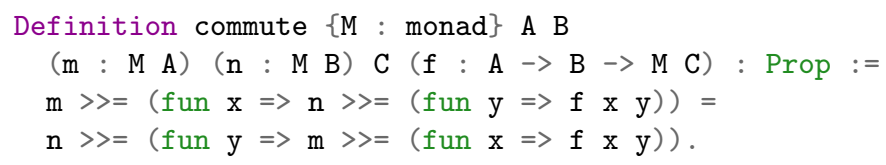

In order to state a generic property of commutativity between nondeterminism and state monads, we first define a predicate that captures syntactically nondeterminism monads. They are written with the following (higher-order abstract [33]) syntax:

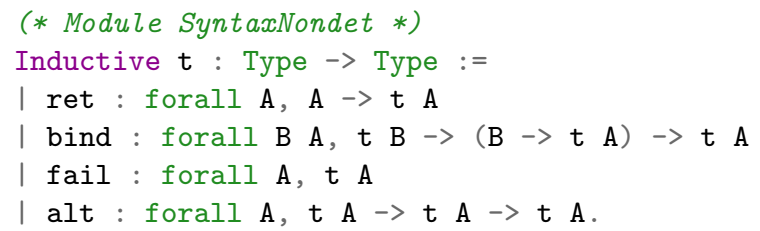

Let denote be a function that turns the above syntax into the corresponding monadic computation:

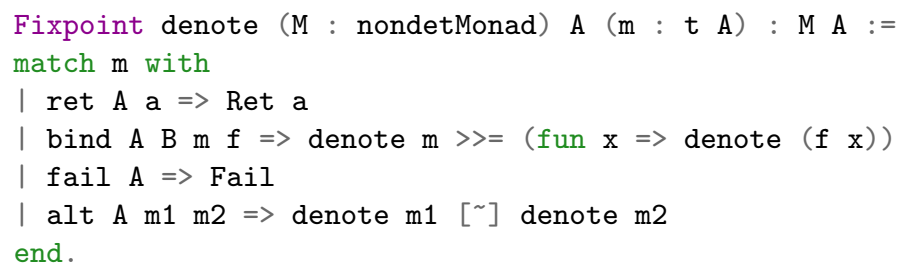

Using above definitions, we can write a predicate that captures computations in a nondetStateMonad that are actually just computations in a nondetMonad:

Definition nondetState_sub S (M : nondetStateMonad S) A (n: M A) :=

$\{\mathrm{m} \mid$ denote $\mathrm{m}=\mathrm{n}\}$.

Eventually, it becomes possible to prove by induction on the syntax that two computations $\mathrm{m}$ and $\mathrm{n}$ using both state and choice commute when $\mathrm{m}$ actually does not use the state effects:

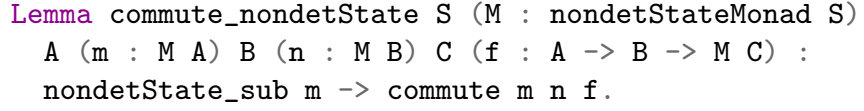

\subsection{Equivalence between Operational and Denotation Semantics}

We consider a small imperative language with a state and an operator to generate events. We equip this language with a small-step semantics and a denotational 
semantics using stateTraceMonad (Sect. 3.3), and prove that both semantics are equivalent. We will see that we need an induction on the syntax to prove this equivalence.

Here follows the (higher-order abstract) syntax of our imperative language:

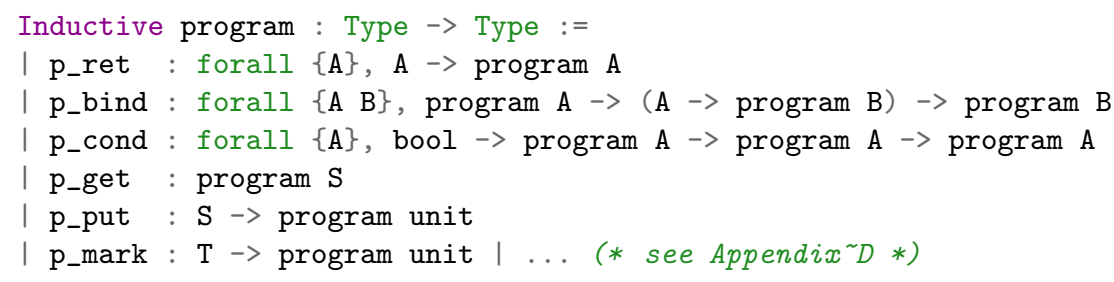

We give our language a small-step semantics specified with continuations in the style of CompCert [5]. We distinguish two kinds of continuations: stop for halting and cont (notation: $\cdot ; \cdot)$ for sequencing:

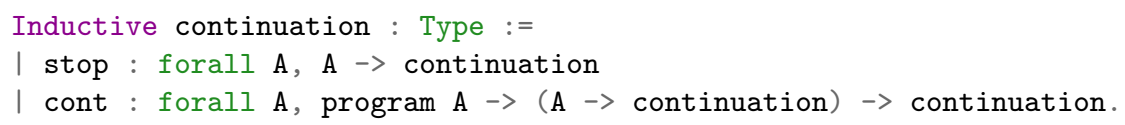

We can then define the ternary relation step that relates a state to the next one and optionally an event:

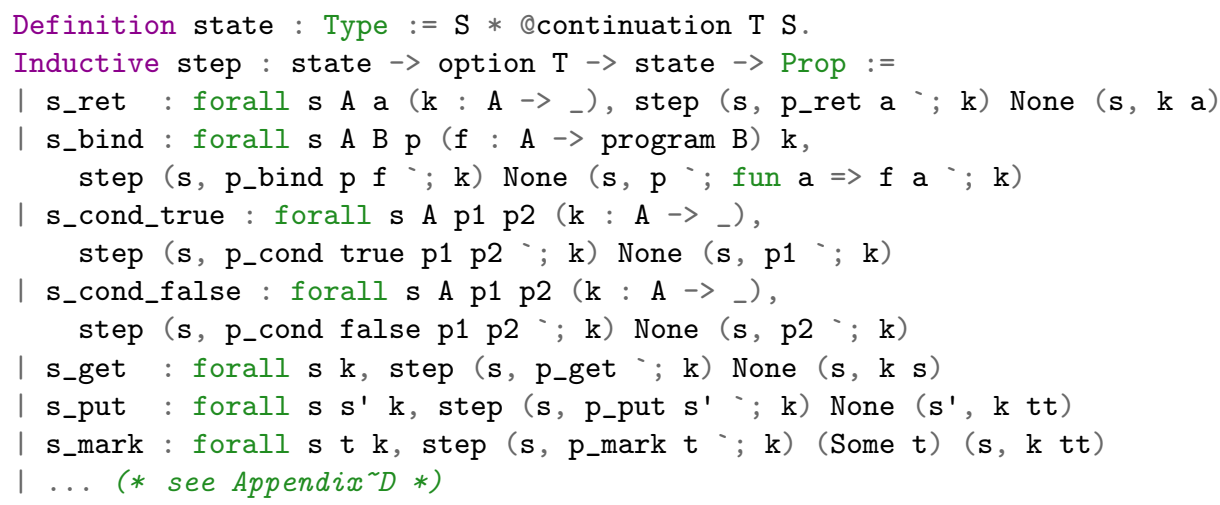

Its reflexive and transitive closure step_star of type state $->$ seq $T->$ state $->$ Prop is defined as one expects. We prove that step is deterministic and that step_star is confluent and deterministic.

We also give our language a denotational semantics using the stateTraceMonad:

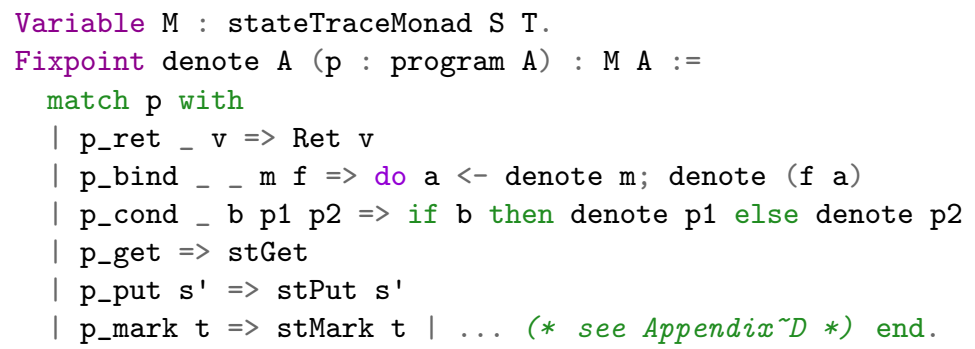


It is important to note here that the operators stGet and stPut can only read and update the state (of type $\mathrm{S}$ ) but not the log of emitted events (of type seq T). Only the operator stMark has access to the list of emitted events but it can neither read nor overwrite it: it can only log a new event to the list.

We proved the correctness and completeness of the small-step semantics step_star w.r.t. the denotational semantics denote [3, file smallstep_monad.v]. For that we use only the equations of the run interface of the state-trace monad (Sect. 3.3). We now come to those parts of the proofs of correctness and completeness that require induction on the syntax. They take the form of two lemmas. Like in the previous section, we introduce a predicate to distinguish the monadic computations that can be written with the syntax of the programming language:

Definition stateTrace_sub A (m : M A) : $=\{p \mid$ denote $p=m\}$.

The first lemma states that once an event is emitted it cannot be deleted:

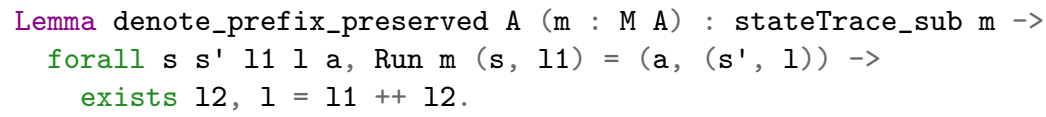

The second lemma states that the remaining execution of a program does not depend on the previously emitted events:

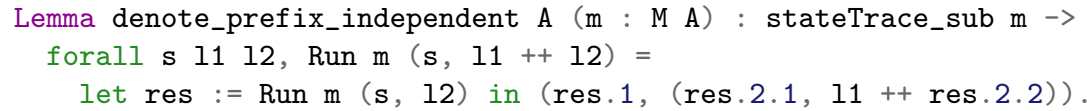

Those are natural properties that ought to be true for any monadic code, and not only the monadic code that results from the denotation of a program. But this is not the case with our monad. Indeed, the interface specifies those operators that should be implemented but does not prevent one to add other operators that might break the above properties of emitted events. This is why we restrict those properties to monadic code using the stateTrace_sub predicate, thus allowing us to prove the two above lemmas by induction on the syntax.

\section{Models of Monads}

Sections 2 and 3 explained how to build a hierarchy of effects. In this section, we complete this formalization by explaining how to provide models, i.e., concrete objects that validate the equational theories. Providing a model amounts to define a function of type Type $\rightarrow$ Type for the base monad and instantiate all the interfaces up to the monad of interest. For illustration, we explain models of state monads and of the probability monad; see [3, file monad_model.v] for simpler models.

\subsection{Models of State Monads}

State-trace Monad. A model for stateTraceMonad (Sect. 3.3) is a function fun $\mathrm{A} \Rightarrow \mathrm{S} *$ seq $\mathrm{T} \rightarrow \mathrm{A} *(\mathrm{~S} *$ seq $\mathrm{T})$. We start by providing the ret and bind operators of the base monad using the constructor Monad_of_ret_bind (Sect. 2.1): 


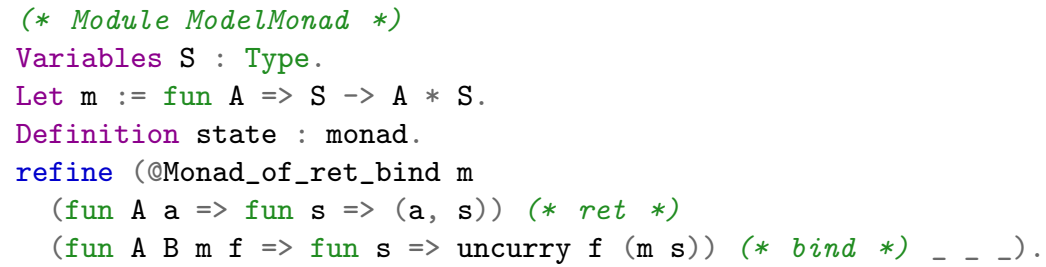

One needs to prove the monad laws to complete this definition. This gives a monad ModelMonad. state upon which we define the get, put, and mark operators:

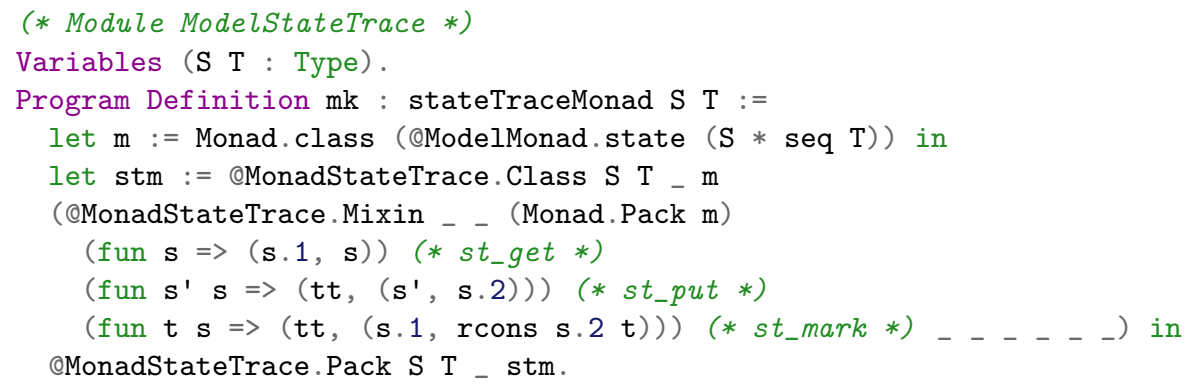

The laws of the state-trace monad are proved automatically by CoQ.

Backtrackable-state. A possible model for nondetStateMonad (Sect. 3.2 is fun $A \Rightarrow S \rightarrow\{$ fset $(A * S)\}$, where $\{$ fset $X\}$ is the type of finite sets over $X$ provided by the FINMAP library. This formalization of finite sets is based on list representations of finite predicates. The canonical representation is chosen uniquely among its permutations. This choice requires the base type $\mathrm{X}$ of $\{\mathrm{f}$ set $\mathrm{X}\}$ to be a choiceType, i.e., a type equipped with a choice function, thus satisfying a form of the axiom of choice. To be able to use the Finmap library, we use a construct (gen_choiceMixin) from the MATHCOMP-ANALYSIS library that can turn any type into a choiceType. We use it to define a model for nondetStateMonad as follows:

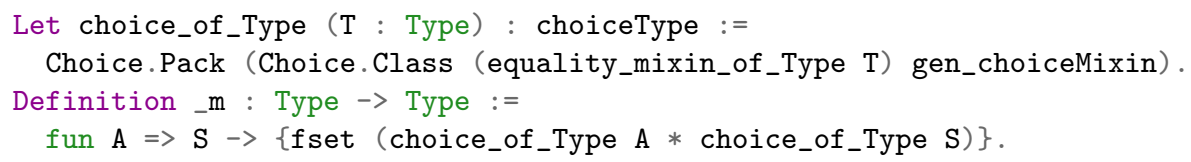

It remains to prove all the algebraic laws of the interfaces up to nondetStateMonad; see [3, file monad_model.v] for details.

\subsection{A Model of the Probability Monad}

A theory of probability distributions provides a model for the probability monad (Sect. 3.4). For this paper, we propose the following definition of probability distribution [4]: 


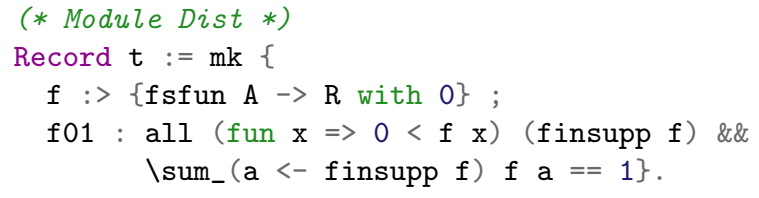

The first field is a finitely-supported function $f$ : it evaluates to 0 outside its support finsupp $\mathrm{f}$. The second field contains proofs that (1) the probability function outputs positive reals and that (2) its outputs sum to 1 . Let Dist be a notation for Dist.t. It has type choiceType $\rightarrow$ choiceType and can therefore be used to build a monad (thanks to choice_of_Type from the previous section).

The bind operator is well-known: given $\mathrm{p}$ : Dist A and $\mathrm{g}: \mathrm{A} \rightarrow$ Dist $\mathrm{B}$, it returns a distribution with probability mass function $b \mapsto \sum_{a \in \operatorname{supp}(\mathrm{p})} \mathrm{p}(a) \cdot \mathrm{g}(a, b)$. This is implemented by the following combinator:

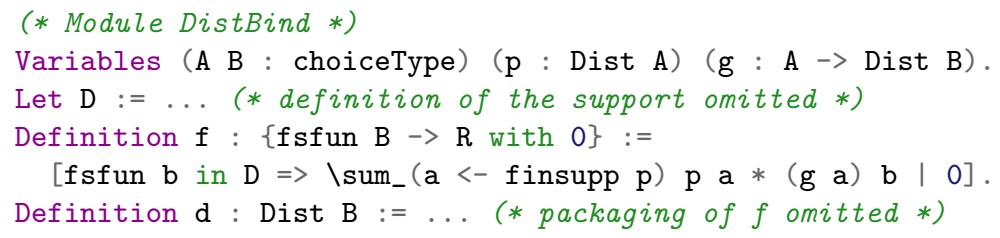

The resulting combinator DistBind.d can be proved to satisfy the monad laws, for example, associativity:

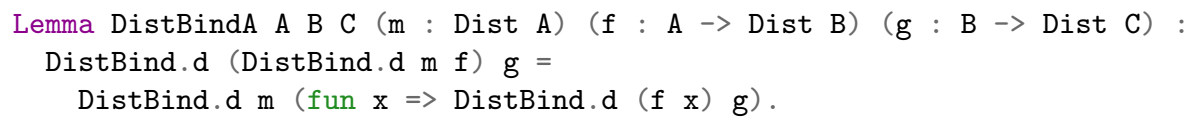

Completing the model with a distribution for the ret operator and the other properties of monads is an exercise.

The last step is to provide an implementation for the interface of the probability monad. The probabilistic choice operator corresponds to the construction of a distribution d from two distributions $\mathrm{d} 1$ and $\mathrm{d} 2$ biased by a probability $\mathrm{p}$ :

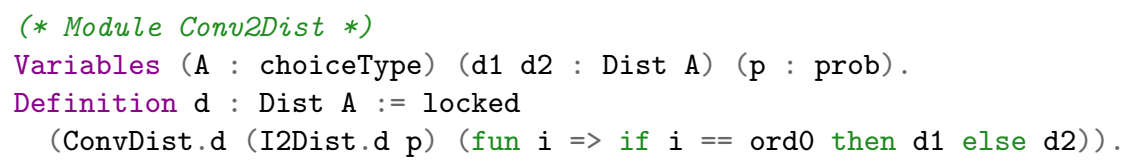

The combinator ConvDist. $d$ is a generalization that handles the combination of any distribution of distributions: it is instantiated here with the binary distribution I2Dist.d $\mathrm{p} 4$. We finally prove that the probabilistic choice $\mathrm{d}$ have the expected properties, for example, skewed commutativity:

Notation $\mathrm{x}<|\mathrm{p}|>\mathrm{y} ":=(\mathrm{d} \mathrm{x} \mathrm{y} \mathrm{p})$. (* probabilistic choice *)

Lemma convC ( $\mathrm{p}$ : prob) $(\mathrm{a} b$ : Dist $\mathrm{A}): \mathrm{a}<|\mathrm{p}|>\mathrm{b}=\mathrm{b}\left\langle\left|{ }^{-} \operatorname{Pr} \mathrm{p} \cdot{ }^{\sim}\right|>\mathrm{a}\right.$. 


\section{Technical Aspects of Formalization in Coq}

About Coq Commands and Tactics. There are several CoQ commands and tactics that are instrumental in our formalization. Most importantly, we use CoQ canonical structures (as implemented by the command Canonical) to implement packed classes (Sect. 2), but also to implement other theories such as probabilities (Sect. 3.4). We already mentioned that the rewrite tactic from SSREFLECT is important to obtain short proof scripts (Sect. 4). We take advantage of the reals of the CoQ standard library which come with automation: the field and lra (linear real/rational arithmetic) tactics are important in practice to compute probabilities (for example in the Monty Hall problem).

About Useful Coq Libraries. We use the SSREFLECT library for lists because it is closer to the Haskell library than the CoQ standard library. It provides Haskell-like notations (e.g., notation for comprehension) and more functions (e.g., allpairs, a.k.a. cp in Haskell). We use the Finmap library of MATHComP for its finite sets (see Sect.6.1). We also benefit from other libraries compatible with МатнСомp to formalize the model of the probability monad [4].

About the Use of Extra Axioms. We use axioms inherited from the MaтнCомрANALYSIS library (they are explained in 2, §5]). More precisely, we use functional extensionality in particular to identify the CoQ functions that appear in the bind operator. We use gen_choiceMixin to turn Types into choiceTypes when constructing models (see Sect. 6). To provide a model for the probability monad (Sect. 6.2), we proposed a type of probability distributions that requires reals to also enjoy an axiom of choice. We also have a localized use of the axiom of proof irrelevance to prove properties of functors [3, file monad.v]. All these axioms make our CoQ environment resemble classical set theory. We choose to go with these axioms because it does not restrict the applicability of our work: equational reasoning does not forbid a classical meta-theory with the axiom of choice.

\section{Related Work}

Formalization of Monads in CoQ. Monads are widely used for modeling programming languages with effects. For instance, Delaware et al. formalize several monads and monad transformers, each one associated with a feature theorem [9]. When monads are combined, those feature theorems can then be combined to prove type soundness. In comparison, the work we formalize here contains more monads and focuses on equational reasoning about concrete programs instead of meta-theory about programming languages.

Monads have been used in CoQ to verify low-level systems [19|20 or for their modular verification [23] based on free monads. Our motivation is similar: enable formal reasoning for effectful programs using monads.

There are more formalizations of monads in other proof assistants. To pick one example that can be easily compared to our mechanization, one can find 
a formalization of the Monty Hall problem in Isabelle [8] (but using the pGCL programming language).

About Monadic Equational Reasoning. Although enabling equational reasoning for reasoning about monadic programs seems to be a natural idea, there does not seem to be much related work. Gibbons and Hinze seem to be the first to synthesize monadic equational reasoning as an approach [1/12 13. This viewpoint is also adopted by other authors 6 6 30 3137.

Applicative functor is an alternative approach to represent effectful computations. It has been formalized in Isabelle/HOL together with the tree relabeling example 24]. This work focuses on the lifting of equations to allow for automation, while our approach is rather the one of small-scale reflection [14]: the construction of a hierarchy backed up by a rich library of definitions and lemmas to make the most out of the rewriting facilities of CoQ.

We extended the hierarchy of Gibbons and Hinze with a state-trace monad with the intent of performing formal verification about programs written with the syntax and semantics of Sect. 5.2. There are actually more topics to explore about the formalization of tracing and monads 34 .

About Formalization Techniques. We use packed classes [11] to formalize the hierarchy of effects. It should be possible to use other techniques. In fact, a preliminary version of our formalization was using a combination of telescopes and canonical structures. It did not suffer major problems but packed classes are more disciplined and are known to scale up to deep hierarchies. CoQ's type classes have been reported to replace canonical structures in many situations, but we have not tested them here.

The problem of rewriting under function abstraction (Sect. 4.3 ) is not specific to monadic equational reasoning. For example, it also occurs when dealing with the big operators of the MATHComp library, a situation for which a forthcoming version of Coq provides automation [27].

\section{Conclusions and Future Work}

We reported on the formalization in the CoQ proof assistant of an extensive hierarchy of effects with their algebraic laws, and its application to monadic equational reasoning. The key technique is the one of packed classes, which allows for the sharing of notations and properties of various monads, enforces modularity by insisting on interfaces, while preserving the ability to provide rigorous models. We also discussed other techniques of practical interest for monadic equational reasoning such as reasoning on the syntax despite dealing with a shallow embedding. As a benchmark, we applied our formalization to several pencil-and-paper proofs and furthermore formalized and proved properties of the semantics of an imperative programming language. Our approach is successful in the sense that our proof scripts closely match their paper-and-pencil counterparts. Our work also led us to revisit existing proofs and extend the hierarchy of effects originally 
proposed by Gibbons and Hinze. We believe that our experiments demonstrate that the formalization of monadic equational reasoning with packed classes and a shallow embedding provides a practical tool for formal verification of effectful programs.

Future Work. We have started the formalization of more examples of monadic equational reasoning [3, branch experiments]: 6] is underway, [10] proposes a sharing monad whose equations seems to call for more syntax reflection and brings to the table the issue of infinite data structures.

In its current state the rewrite_ tactic (Sect. 4.3 is not completely satisfactory. Its main defect is practical: it cannot be chained with the standard rewrite tactic. We defer the design of a better solution to future work because the topic is actually more general (as discussed in Sect. 8).

The main task that we are now addressing is the formalization of the model of the monad that combines probability and nondeterminism. Though wellunderstood [7, its formalization requires a careful formalization of convexity, which is work in progress.

It remains to check whether we can improve the modularity of model construction (or even the extension of the hierarchy) through formalizing other generic methods for combining effects, such as algebraic effects and distributive laws between monads.

Acknowledgements. We acknowledge the support of the JSPS-CNRS bilateral program "FoRmal tools for IoT sEcurity" (PRC2199) and the JSPS KAKENHI Grant Number 18H03204, and thank all the participants of these projects for fruitful discussions. In particular, we thank Jacques Garrigue and Samuel Hym for taking the time to have extended discussions and giving us feedback on drafts of this paper. We also thank Cyril Cohen and Shinya Katsumata for comments about the formalization of monads.

\section{A The Choice Monad}

The following excerpt from the source code 3 ] corresponds to the choice monad first mentioned in Sect. 2.2.

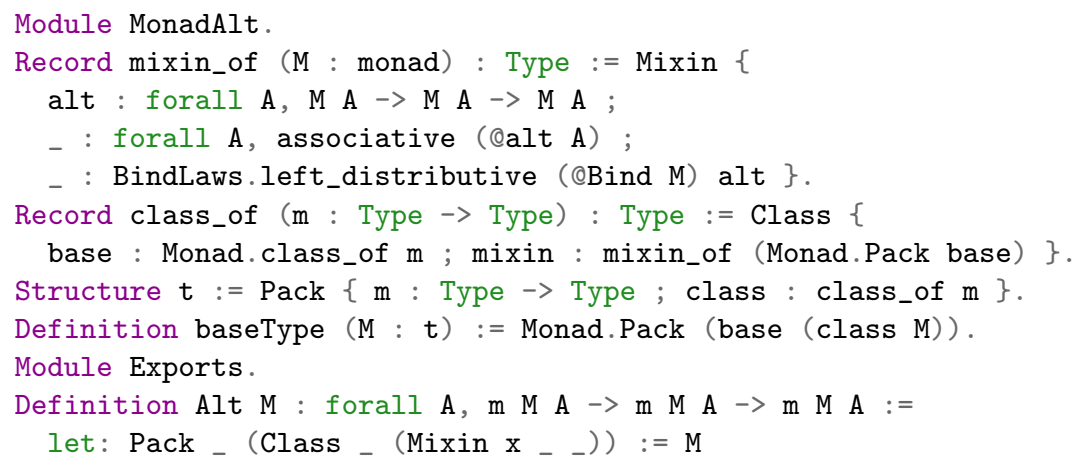




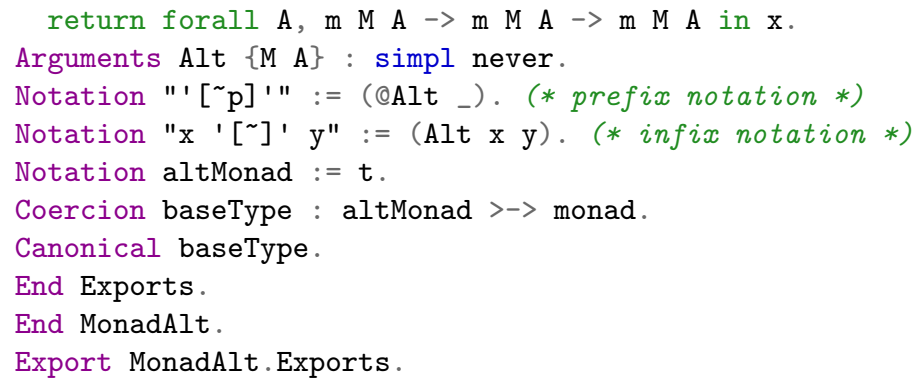

\section{B Generic Algebraic Laws}

The algebraic laws used in this paper are instances of generic definitions with self-explanatory names. Table 1 summarizes the laws defined in SSREFLECT (file ssrfun.v from the standard distribution of CoQ). Table 2 summarizes the laws introduced in this paper. The CoQ definitions are available online [3].

Table 1. Algebraic laws defined in SSREFLECT

\begin{tabular}{|l|l|}
\hline associative op & $\forall x, y, z . x$ op $(y \circ \mathrm{op} z)=(x \circ \mathrm{op} y)$ op $z$ \\
left_id e op & $\forall x . e$ op $x=x$ \\
right_id e op & $\forall x . x$ op $e=x$ \\
left_zero z op & $\forall x . z$ op $x=z$ \\
idempotent op & $\forall x . x$ op $x=x$ \\
\hline
\end{tabular}

\section{Summary of Monads and their Algebraic Laws}

Table 3 summarizes the structures and the algebraic laws that we formalize and explain in this paper. Precise CoQ definitions are available online [3].

\section{Details about the Imperative Language from Sect. 5.2}

For the sake of completeness, we provide the definition of the syntax (program) and semantics (operational step and denotational denote) of the imperative language of Sect. 5.2 where we omitted looping constructs to help reading:

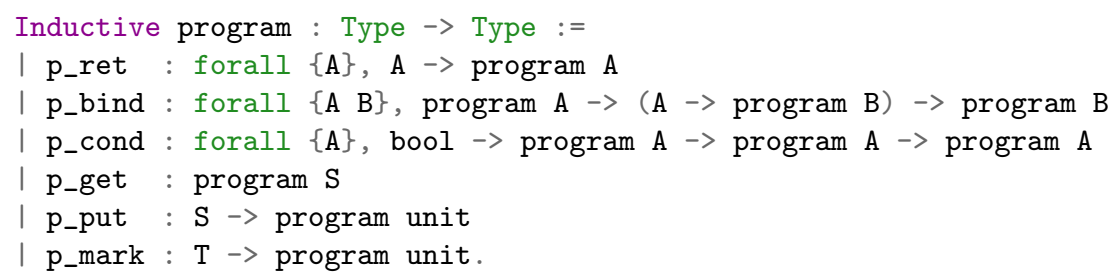


Table 2. Algebraic laws defined in this paper

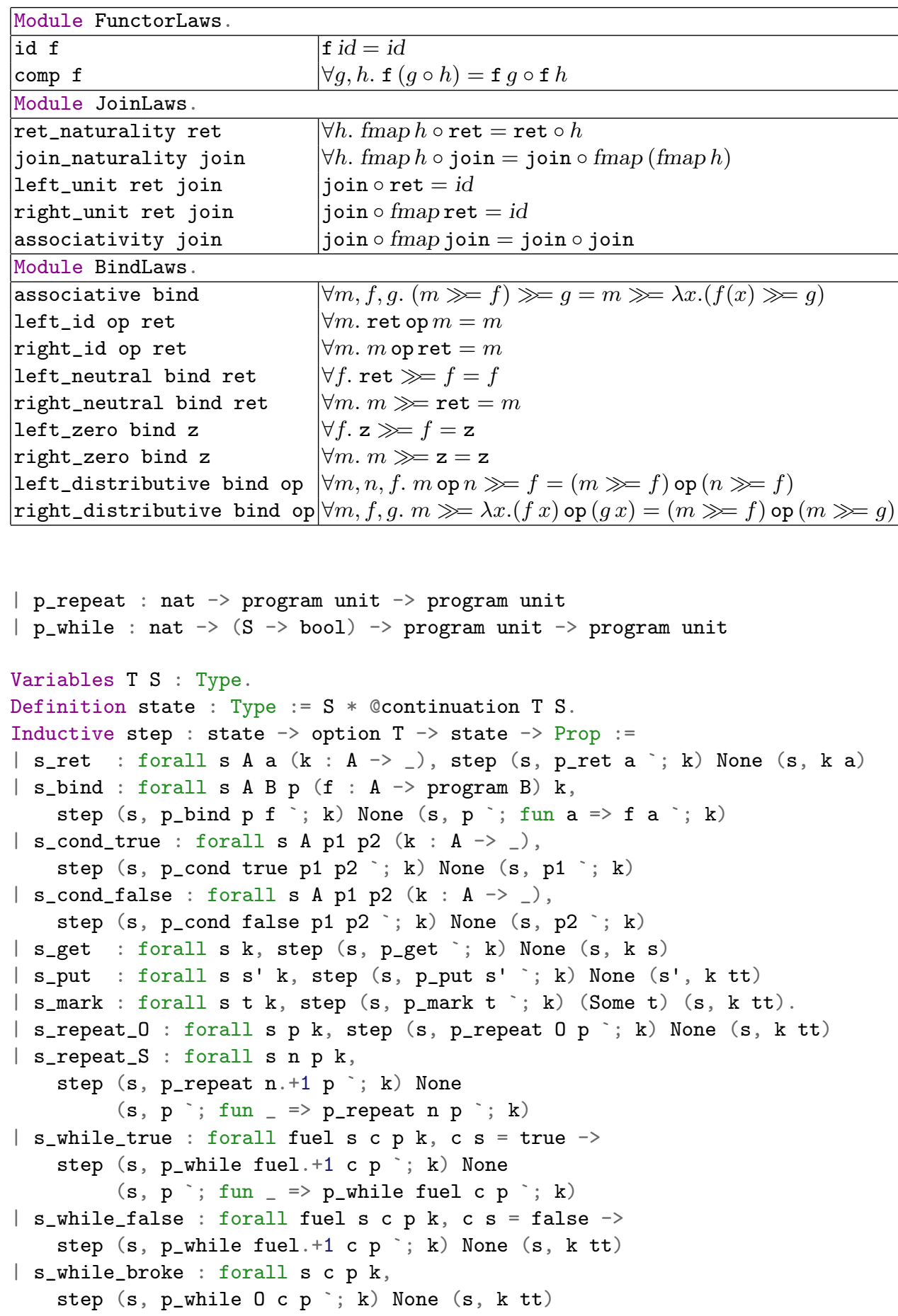




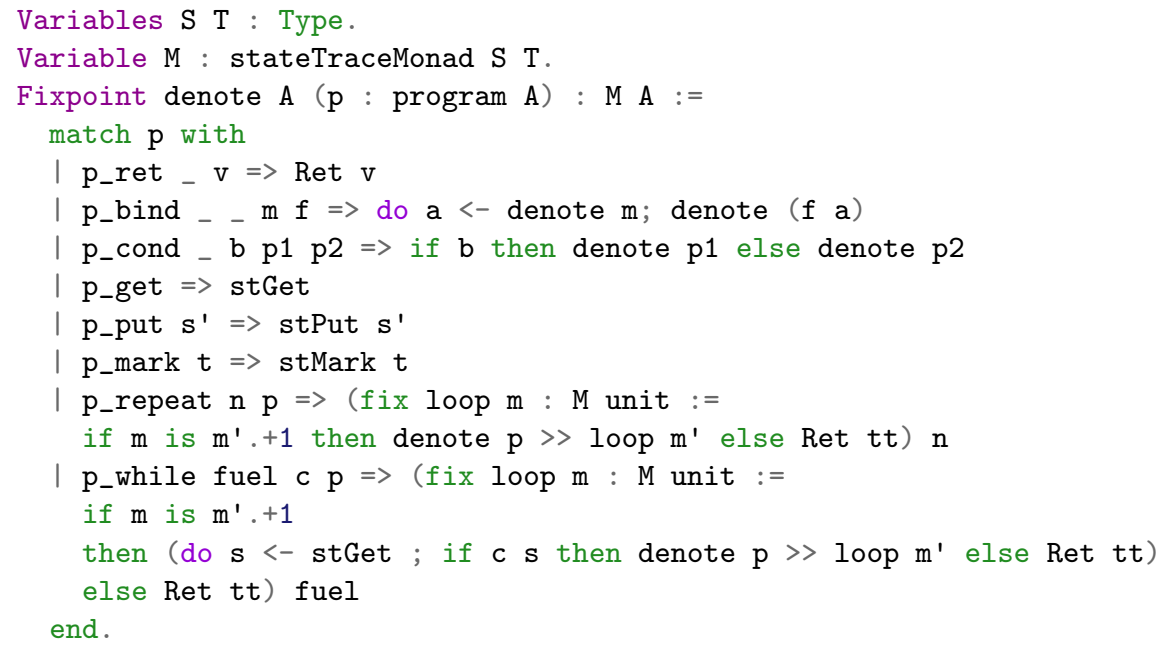

\section{References}

1. Abou-Saleh, F., Cheung, K.H., Gibbons, J.: Reasoning about probability and nondeterminism. In: Workshop on probabilistic programming semantics, St. Petersburg, FL, USA, January 23, 2016 (Jan 2016)

2. Affeldt, R., Cohen, C., Rouhling, D.: Formalization techniques for asymptotic reasoning in classical analysis. J. Formaliz. Reason. 11(1), 43-76 (2018)

3. Affeldt, R., Garrigue, J., Nowak, D., Saikawa, T.: A Coq formalization of monadic equational reasoning. https://github.com/affeldt-aist/monae (2018)

4. Affeldt, R., Hagiwara, M., Sénizergues, J., Garrigue, J., Sakaguchi, K., Asai, T., Saikawa, T., Obata, N.: A Coq formalization of information theory and linear error-correcting codes. https://github.com/affeldt-aist/infotheo (2018)

5. Appel, A.W., Blazy, S.: Separation logic for small-step Cminor. In: 20th Int. Conf. on Theorem Proving in Higher Order Logics (TPHOLs 2007). Lecture Notes in Computer Science, vol. 4732, pp. 5-21. Springer (2007)

6. Chen, Y., Hong, C., Lengál, O., Mu, S., Sinha, N., Wang, B.: An executable sequential specification for spark aggregation. In: 5th Int. Conf. on Networked Systems (NETYS 2017) Marrakech, Morocco, May 17-19, 2017. Lecture Notes in Computer Science, vol. 10299, pp. 421-438 (2017)

7. Cheung, K.H.: Distributive Interaction of Algebraic Effects. Ph.D. thesis, Merton College, University of Oxford (2017)

8. Cock, D.: Verifying probabilistic correctness in Isabelle with pGCL. In: 7th Systems Software Verification, Sydney, Australia. pp. 1-10 (Nov 2012)

9. Delaware, B., Keuchel, S., Schrijvers, T., d. S. Oliveira, B.C.: Modular monadic meta-theory. In: ACM SIGPLAN Int. Conf. on Functional Programming (ICFP 2013), Boston, MA, USA, September 25-27, 2013. pp. 319-330 (2013)

10. Fischer, S., Kiselyov, O., Shan, C.: Purely functional lazy nondeterministic programming. J. Funct. Program. 21(4-5), 413-465 (2011)

11. Garillot, F., Gonthier, G., Mahboubi, A., Rideau, L.: Packaging mathematical structures. In: 22nd Int. Conf. on Theorem Proving in Higher Order Logics (TPHOLs 2009), Munich, Germany, August 17-20, 2009. Lecture Notes in Computer Science, vol. 5674, pp. 327-342. Springer (2009) 
12. Gibbons, J.: Unifying theories of programming with monads. In: 4th Int. Symp. on Unifying Theories of Programming (UTP 2012), Paris, France, August 27-28, 2012, Revised Selected Papers. Lecture Notes in Computer Science, vol. 7681, pp. 23-67. Springer (2013)

13. Gibbons, J., Hinze, R.: Just do it: simple monadic equational reasoning. In: 16th ACM SIGPLAN Int. Conf. on Functional Programming (ICFP 2011), Tokyo, Japan, September 19-21, 2011. pp. 2-14. ACM (2011)

14. Gonthier, G., Mahboubi, A.: An introduction to small scale reflection in Coq. J. Formaliz. Reasoning 3(2), 95-152 (2010)

15. Gonthier, G., Tassi, E.: A language of patterns for subterm selection. In: 3rd Int. Conf. on Interactive Theorem Proving (ITP 2012), Princeton, NJ, USA, August 13-15, 2012. Lecture Notes in Computer Science, vol. 7406, pp. 361-376. Springer (2012)

16. Greenaway, D.: Automated Proof-Producing Abstraction of C Code. Ph.D. thesis, University of New South Wales, Sydney, Australia (Jan 2015)

17. Hirschowitz, A., Maggesi, M.: Modules over monads and initial semantics. Inf. Comput. 208(5), 545-564 (2010)

18. Jacobs, B.: Convexity, duality and effects. In: IFIP TCS. IFIP Advances in Information and Communication Technology, vol. 323, pp. 1-19. Springer (2010)

19. Jomaa, N., Nowak, D., Grimaud, G., Hym, S.: Formal proof of dynamic memory isolation based on MMU. Sci. Comput. Program. 162, 76-92 (2018)

20. Jomaa, N., Torrini, P., Nowak, D., Grimaud, G., Hym, S.: Proof-oriented design of a separation kernel with minimal trusted computing base. In: 18th Int. Work. on Automated Verification of Critical Systems (AVOCS 2018), July 2018, Oxford, UK. Electronic Communications of the EASST Open Access Journal (2018)

21. Jones, M.P., Duponcheel, L.: Composing monads. Tech. Rep. YALEU/DCS/RR1004, Yale University (Dec 1993)

22. King, D.J., Wadler, P.: Combining monads. In: Functional Programming. pp. 134143. Workshops in Computing, Springer (1992)

23. Letan, T., Régis-Gianas, Y., Chifflier, P., Hiet, G.: Modular verification of programs with effects and effect handlers in Coq. In: 22nd Int. Symp. on formal methods (FM 2018), Oxford, UK (Jul 2018)

24. Lochbihler, A., Schneider, J.: Equational reasoning with applicative functors. In: 7th Int. Conf. on Interactive Theorem Proving (ITP 2016), Nancy, France, August 22-25, 2016. Lecture Notes in Computer Science, vol. 9807, pp. 252-273. Springer (2016)

25. Mac Lane, S.: Categories for the Working Mathematician, Graduate Texts in Mathematics, vol. 5. Springer-Verlag, New York, second edn. (1998)

26. Mahboubi, A., Tassi, E.: Mathematical Components. Available at https:// math-comp.github.io/mcb/ (2016), with contributions by Yves Bertot and Georges Gonthier. Version of 2018/08/11.

27. Martin-Dorel, E., Tassi, E.: SSReflect in Coq 8.10. In: The Coq Workshop 2019, Portland, OR, USA, September 8, 2019. pp. 1-2 (Sep 2019)

28. Moggi, E.: Computational lambda-calculus and monads. In: LICS. pp. 14-23. IEEE Computer Society (1989)

29. $\mathrm{Mu}$, S.C.: Functional pearls, reasoning and derivation of monadic programs, a case study of non-determinism and state (Jul 2017), draft. Available at http://flolac. iis.sinica.edu.tw/flolac18/files/test.pdf (last access: 2019/07/10)

30. Mu, S.C.: Calculating a backtracking algorithm: An exercise in monadic program derivation. Tech. Rep. TR-IIS-19-003, Institute of Information Science, Academia Sinica (Jun 2019) 
31. $\mathrm{Mu}$, S.C.: Equational reasoning for non-determinism monad: A case study of Spark aggregation. Tech. Rep. TR-IIS-19-002, Institute of Information Science, Academia Sinica (Jun 2019)

32. $\mathrm{Mu}, \mathrm{S} ., \mathrm{Ko}, \mathrm{H} .$, Jansson, P.: Algebra of programming in Agda: Dependent types for relational program derivation. J. Funct. Program. 19(5), 545-579 (2009)

33. Pfenning, F., Elliott, C.: Higher-order abstract syntax. In: ACM SIGPLAN Conference on Programming Language Design and Implementation (PLDI 1988), Atlanta, GA, USA, June 22-24, 1988. pp. 199-208. ACM (1988)

34. Piróg, M., Gibbons, J.: Tracing monadic computations and representing effects. In: 4th Workshop on Mathematically Structured Functional Programming (MSFP 2012), Tallinn, Estonia, March 25, 2012. EPTCS, vol. 76, pp. 90-111 (2012)

35. Plotkin, G.D., Power, J.: Notions of computation determine monads. In: FoSSaCS. Lecture Notes in Computer Science, vol. 2303, pp. 342-356. Springer (2002)

36. Pretnar, M.: An introduction to algebraic effects and handlers (invited tutorial paper). Electr. Notes Theor. Comput. Sci. 319, 19-35 (2015)

37. Shan, C.C.: Equational reasoning for probabilistic programming. In: POPL 2018 TutorialFest (Jan 2018)

38. Varacca, D., Winskel, G.: Distributing probability over non-determinism. Mathematical Structures in Computer Science 16(1), 87-113 (2006)

39. Voevodsky, V., Ahrens, B., Grayson, D., et al.: UniMath-a computer-checked library of univalent mathematics. Available at https://github.com/UniMath/ UniMath

40. Wadler, P.: Comprehending monads. In: LISP and Functional Programming. pp. 61-78 (1990) 
Table 3. Monads Defined in this Paper and the Algebraic Laws They Introduce

\begin{tabular}{|c|c|c|}
\hline Structure & Operators & Equations \\
\hline functor $(2.1)$ & Fun/\# & functor_id, functor_o \\
\hline \multirow[t]{3}{*}{ monad (\$2.1) } & Ret & ret_naturality \\
\hline & Join & $\begin{array}{l}\text { join_naturality, joinretM (left unit), } \\
\text { joinMret (right unit), joinA (associativity) }\end{array}$ \\
\hline & Bind $/>>=/>>$ & $\begin{array}{l}\text { bindretf (left neutral), bindmret (right neutral), } \\
\text { bindA (associativity) }\end{array}$ \\
\hline failMonad ( 2.2 & Fail & bindfailf (fail left-zero of bind) \\
\hline altMonad ( A) & Alt $/[\sim] /[\sim \mathrm{p}]$ & $\begin{array}{l}\text { alt_bindDl (bind left-distributes over choice), } \\
\text { altA (associativity) }\end{array}$ \\
\hline nondetMonad ( 2.2 & & altmfail (right-id), altfailm (left-id) \\
\hline exceptMonad ( 3.1 & Catch & $\begin{array}{l}\text { catchfailm (left-id), catchmfail (right-id), } \\
\text { catchA (associativity), catchret (left-zero) }\end{array}$ \\
\hline stateMonad ( $(3.2)$ & Get, Put & putget, getputskip, putput, getget \\
\hline runMonad ( 3.2$)$ & Run & runret, runbind \\
\hline stateRunMonad (\$3.2) & & runget, runput \\
\hline nondetStateMonad 3.2 & & $\begin{array}{l}\text { bindmfail (right-zero), } \\
\text { alt_bindDr (bind right-distributes over choice) }\end{array}$ \\
\hline traceMonad ( 3.3 & Mark & \\
\hline \multirow[t]{3}{*}{ stateTraceMonad 3.3} & stGet & st_getget \\
\hline & stPut & st_putput, st_putget, st_getputskip \\
\hline & stMark & st_putmark, st_getmark \\
\hline traceRunMonad ( 3.3 & & runmark \\
\hline stateTraceRunMonad ( 3.3$)$ & & runstget, runstput, runstmark \\
\hline probMonad (\$3.4) & Choice & $\begin{array}{l}\text { choicemm (idempotence), } \\
\text { choice0, choice1 (identity laws), } \\
\text { choiceA (quasi associativity), } \\
\text { choiceC (skewed commutativity), } \\
\text { prob_bindDl (bind left-distributes over choice) }\end{array}$ \\
\hline altCIMonad ( 3.5 & & altmm (idempotence), altC (commutativity) \\
\hline \multicolumn{3}{|l|}{ nondetCIMonad (3.5) } \\
\hline freshMonad $(3.5)$ & Fresh & \\
\hline failFreshMonad (3.5) & Distinct & $\begin{array}{l}\text { failfresh_bindmfail (fail right-zero of bind) } \\
\text { bassert (Distinct M) \o Symbols = Symbols }\end{array}$ \\
\hline arrayMonad $(\$ 3.5)$ & $\begin{array}{l}\text { aGet i, } \\
\text { aPut i s }\end{array}$ & $\begin{array}{l}\text { aputput, aputget, agetputskip, agetget, } \\
\text { agetC, aputC, aputgetC }\end{array}$ \\
\hline probDrMonad ( 3.5$)$ & & prob_bindDr (bind right-distributes over choice) \\
\hline altProbMonad 3.5 & & $\begin{array}{l}\text { choiceDr (probabilistic choice right-distributes } \\
\text { over nondeterministic choice) }\end{array}$ \\
\hline exceptProbMonad (\$3.5) & & catchDl (catch left-distributes over choice) \\
\hline
\end{tabular}

